\title{
Parkinson Disease-Modification Encompassing Rotenone and 6-Hydroxydopamine Neurotoxicity by the Microtubule-Protecting Drug Candidate SKIP
}

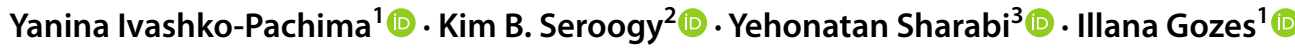 \\ Accepted: 17 June 2021 / Published online: 21 July 2021 \\ (c) The Author(s), under exclusive licence to Springer Science+Business Media, LLC, part of Springer Nature 2021
}

\begin{abstract}
Encompassing live cell imaging and morphometrics at the microscopical level, we showed here, for the first time, protection of neuronal-like cells by the novel drug candidate, SKIP, against the Parkinson's disease-related neurotoxin, rotenone. Mechanistically, rotenone disrupted microtubule dynamics, which SKIP partially repaired through microtubule end-binding proteins, coupled with increasing neurite branch length. Given the previous association of rotenone toxicity with increased dopaminergic cell death hallmarking Parkinson's disease, we chose an established rat model of 6-hydroxydopamine (6-OHDA) toxicity to initially evaluate SKIP in vivo. SKIP pretreatment showed protection against nigral dopaminergic cell degeneration and improved motor behavior in the forelimb asymmetry test. With Parkinson's disease being a major neurodegenerative disorder, afflicting millions of people globally, and with disease modification challenges, SKIP may hold promise for future therapeutic development.
\end{abstract}

\section{Introduction}

Neurodegenerative diseases in general and Parkinson disease (PD) in particular are posing a significant challenge to public health. Here, we focus on intervention and prevention or modification of the disease process by targeting fundamental brain protection. Twenty years ago, the Gozes laboratory discovered a new protein and named it activitydependent neuroprotective protein (ADNP) (Bassan et al. 1999). ADNP (Zamostiano et al. 2001) has transcription

Illana Gozes and Yehonatan Sharabi contributed equally on this work.

Yehonatan Sharabi

Illana Gozes

igozes@tauex.tau.ac.il

1 Elton Laboratory for Molecular Neuroendocrinology, Department of Human Molecular Genetics and Biochemistry, Sackler Faculty of Medicine, Adams Super Center for Brain Studies and Sagol School of Neuroscience, Tel Aviv University, 69978 Tel Aviv, Israel

2 Department of Neurology, College of Medicine, University of Cincinnati, Cincinnati, OH 45267, USA

3 Sheba Medical Center and Sackler Faculty of Medicine, Tel Aviv University, 69978 Tel Aviv, Israel factor properties regulating $>400$ genes during development (Mandel et al. 2007) and controls microtubules (MTs). As such, ADNP is essential for brain formation and function (Pinhasov et al., 2003; Amram et al. 2016). ADNP contains a NAPVSIPQ (NAP) domain, including the SxIP motif, which interacts with MT end-binding proteins (EB1, EB3) enhancing/replacing ADNP-MT interactions, thereby regulating axonal transport and synapse formation $(\mathrm{Oz}$ et al. 2014; Amram et al., 2016; Hacohen-Kleiman et al. 2018). ADNP deficiency in mice results in vocalization, cognitive, and motor impediments and tau pathology (Vulih-Shultzman et al. 2007; Hacohen-Kleiman et al. 2018). NAP treatment corrects these abnormalities (Vulih-Shultzman et al. 2007; Hacohen-Kleiman et al. 2018; Sragovich et al. 2019). Mechanistically, NAP enhances dynamic tau interactions with MTs through MT end-binding proteins (Ivashko-Pachima et al. 2017; 2021a; 2021b). The Gozes laboratory recently designed SKIP (based on the SxIP MT-interacting motif in NAP). SKIP interacts with the NAP motif in ADNP to protect MTs and provides neuroprotection in the Adnp-deficient mouse, which mimics the autistic/intellectual disabilityassociated ADNP syndrome (Amram et al. 2016; HacohenKleiman et al. 2018; Ivashko-Pachima and Gozes 2019a).

A potentially important link between ADNP and PD, a neurodegenerative disease rather than a disease of neuronal development (like the ADNP syndrome), is that brain tissue 
from PD patients exhibits markedly reduced ADNP protein levels in neuromelanin-containing nigral neurons. Reduced ADNP levels occur early in PD (Chu et al. 2016) before reductions in catecholaminergic innervation (as indicated by tyrosine hydroxylase) are detected. Interestingly, our most recent findings (Hadar et al. 2021) identified two key interaction sites for ADNP and sirtuin 1 (SIRT1, a protein positively associated with aging, that in turn is linked with $\mathrm{PD})$. One site is at the EB1 and EB3/tau level, with EB1/ EB3 serving as amplifiers for MT dynamics, synapse formation, axonal transport and protection against tauopathy. The second is on the DNA/chromatin site, with yin yang 1 (YY1), histone deacetylase 2 (HDAC2) and ADNP sharing a DNA-binding motif and regulating SIRT1, ADNP and EB1 (also known as MAPRE1). This interaction was linked to sex- and age-dependent altered histone modification, associated with ADNP/SIRT1/WD repeat-containing protein 5 (WDR5), that mediates the assembly of histone modification complexes. In postmortem brains, we demonstrated a tight correlation for the gene transcripts described above, including related gene products. When analyzing PD brains in comparison to controls, the correlation was maintained except for the most affected substantia nigra, suggesting an important role for ADNP in PD (Hadar et al. 2021).

Furthermore, ADNP levels are also decreased in a rat model of PD (Chu et al. 2016) based on viral over-expression of human wild-type $\alpha$-synuclein (AS, the major risk gene for PD), establishing a potential association between ADNP and AS-PD. Thus, downregulation of ADNP might contribute to dopaminergic neurodegeneration via AS in PD. Complementing these findings, in vitro NAP protects against (1) dopamine (DA) and 6-hydroxydopamine (6-OHDA) toxicity in rat pheochromocytoma PC12 cells and human neuroblastoma cell lines (an established model for PD) (Offen et al. 2000), (2) AS oligomerization/aggregation (Melo et al. 2017), (3) PD mitochondria-inhibited transport, (4) PD-associated MT dysfunction, and (5) reduced autophagic flux (Esteves et al. 2014). NAP also protects against MT dysfunction/tauopathy in an AS PD mouse model (Fleming et al. 2011; Magen et al. 2014). Compared to NAP, SKIP offers an advantage as a potential disease-modifying treatment of PD in that it is a smaller molecule consisting of only 4 amino acids and is therefore more likely to cross the blood-brain barrier in equal or potentially higher amounts (Gozes et al. 2005; Gozes 2020; Amram et al. 2016) and protect against axonal deficits as evidenced in the Adnp-deficient mice (Amram et al. 2016; Ivashko-Pachima and Gozes 2019a).

The movement deficits defining PD are well known to result from drastic depletion of DA in the nigrostriatal system (Kish et al. 1988). According to the catecholaldehyde hypothesis, cytoplasmic accumulation of the catecholaldehyde 3,4-dihydroxyphenylacetaldehyde (DOPAL), the immediate product of the action of monoamine oxidase (MAO) on cytoplasmic DA, is central to the degenerative process (Burke et al. 2003). In PD, three abnormalities tend to build up cytoplasmic DOPAL: (1) decreased vesicular sequestration of cytoplasmic catecholamines, (2) decreased metabolism of DOPAL by aldehyde dehydrogenase (ALDH) (Goldstein et al. 2013), (3) decreased delivery of vesicles to the terminals mediated by MT depolymerization. Postmortem studies analyzing catechol patterns in putamen tissue from PD patients have confirmed this "triple hit." Importantly, the extent of catecholamine depletion in PD is greater than can be accounted for by denervation alone. The implication is that there is a population of dysfunctional neurons that are "sick but not dead" and thus potentially salvageable.

In summary, PD is characterized by DAergic neuronal death in the midbrain and subsequent impairment in motor functions. Inhibition of mitochondrial complex I of the mitochondrial respiratory chain (Schapira 2008) and/or defective regulation of MTs (Choi et al. 2011) have been proposed as possible mechanisms of this selective neurodegeneration.

Rotenone is a well-known pesticide/insecticide that inhibits mitochondrial complex I and also depolymerizes MTs (Srivastava et al. 2007). Thus, combining catecholamines and MTs in PD toxicity, several studies have examined mechanisms besides complex 1 inhibition by which rotenone induces neurotoxicity. One of these is MT assembly (Srivastava and Panda 2007; Passmore et al. 2017). Other studies (Ren and Feng 2007) showed that rotenone is toxic to DA neurons in midbrain cultures and that the MTstabilizing drug taxol mitigates this effect. MT depolymerization disrupts vesicular transport along the MT shaft and causes accumulation of DA vesicles in the soma. This leads to increased oxidative stress due to oxidation of cytosolic DA leaking from vesicles. Concurrently, decreased delivery of vesicles and vesicle-associated proteins to the terminals would be expected to limit vesicular sequestration of locally synthesized DA. These results suggest that MT depolymerization induced by toxins such as rotenone participate in the vulnerability of DA neurons at both the cell soma and the terminals (Ren et al. 2003, 2005; Ren and Feng 2007).

In cell culture models for measuring MT dynamics (IvashkoPachima et al. 2017, 2019b, 2021b), SKIP protects against MT disruption by zinc intoxication associated with Alzheimer's disease (Ivashko-Pachima and Gozes 2019a). Here, we asked whether this protection by SKIP is extended in vitro to protection against rotenone and in vivo to attenuate nigrostriatal 6-OHDA neurotoxicity. We hypothesized that SKIP protects catecholaminergic neurons and ameliorates the neurobehavioral, neurochemical, and neuropathological effects of rotenone and/or 6-OHDA toxicity. 


\section{Materials and Methods}

\section{Materials}

SKIP (under patent protection, Ramot@ Tel Aviv University, I.G., inventor) was custom synthesized by Hai Laboratories.

\section{Methods}

Cell Cultures. Human neuroblastoma SH-SY5Y cells (passage numbers 14-16, ECACC, Public Health England, Porton Down, Salisbury, UK) and mouse neuroblastoma N1E115 cells (passage numbers 16-18, ATCC, Bethesda, MD, USA) were plated onto 96-well plates at a concentration of 3000 cells/well and then differentiated during a week with retinoic acid $(10 \mu \mathrm{M})$ or with reduced FBS $(2 \%)$ and DMSO $(1.25 \%)$, respectively.

Cell Viability Assay (XTT). On the experimental day, differentiated SH-SY5Y cells were treated with 1, 10, and $100 \mu \mathrm{M}$ of rotenone during 24 or $48 \mathrm{~h}$. Since the rotenone stock had been originally diluted into DMSO, "Control" groups were performed with equal volume of DMSO alone. Results of mitochondrial activity (cell viability) were obtained by the XTT colorimetric assay, according to the manufacturer's instructions (XTT-based cell proliferation kit, Biological Industries, Beit Haemek, Israel). Statistical analysis was performed by one-way ANOVA, Tukey, or LSD HSD (IBM SPSS 23): * $p<0.05$, *** $p<0.001$.

Cell Morphology and Polymerized vs. Soluble Microtubule Assay. Human neuroblastoma SH-SY5Y cells were plated onto $10-\mathrm{cm}$ plates and differentiated as described above. Cells were treated with $1 \mu \mathrm{M}$ or $10 \mu \mathrm{M}$ rotenone during 2 or $24 \mathrm{~h}$ with or without SKIP at $10^{-15} \mathrm{M}, 10^{-12} \mathrm{M}$, or $10^{-9} \mathrm{M}$. Cell branch (neurite) length was measured by Fiji (NIH) (Schindelin et al. 2012), and data were analyzed by one-way ANOVA, Tukey HSD (IBM SPSS 23): * $p<0.05$, $* * p<0.01, * * * p<0.001$.

After pictures of cell morphology had been taken, cells were harvested, and lysates were separated into polymerized (P) and soluble (S) protein fractions. Equal aliquots of each pair ( $\mathrm{P}$ and $\mathrm{S}$ ) were resolved on adjacent lanes by SDS polyacrylamide gel electrophoresis (Oz et al. 2014). The blots were probed with tubulin antibodies (alpha-tubulin, T6199, Sigma, Rehovot, Israel). The intensity of each band was quantified by Fiji, and the polymerized ratios were calculated by dividing the intensity value of the polymerized band over the sum of intensity values of polymerized and soluble bands $[\mathrm{P} /(\mathrm{S}+\mathrm{P})]$. Data were statistically analyzed by one-way ANOVA, LSD HSD (IBM SPSS 23) ( $n=2 /$ group): $* p<0.05$.
Time-Lapse Live Cell Imaging of EB3 Comet-like Structures. Forty-eight hours before live imaging, differentiated N1E-115 cells were transfected with $1 \mu \mathrm{g}$ of EB3-RFP-expressing plasmid (Ivashko-Pachima et al. 2017). On the experimental day, differentiated N1E-115 cells were treated for $1 \mathrm{~h}$ with rotenone at final concentrations of $0.1,1$, and $10 \mu \mathrm{M}$, with or without SKIP at $10^{-15} \mathrm{M}, 10^{-12} \mathrm{M}$, and $10^{-9} \mathrm{M}$. During time-lapse imaging, N1E-115 cells were incubated at $37^{\circ} \mathrm{C}$ with a $5 \% \mathrm{CO}_{2} / 95 \%$ air mixture in a thermostatic chamber placed on the stage of a Leica TCS SP5 confocal microscope (objective 100 (PL Apo) oil immersion, NA 1.4). Time-lapse images were automatically captured every $3 \mathrm{~s}$ for $1 \mathrm{~min}$, using the Leica LAS AF software (Leica Microsystems, Wetzlar, Germany). Data were collected and analyzed by Imaris software (Bitplane, Concord, MA, USA). Statistical analysis was done by one-way ANOVA, Tukey HSD (IBM SPSS 23): * $p<0.05, * * p<0.01, * * * p<0.001$.

In vivo SKIP Injections and Partial Lesion 6-OHDA Rat Model. Adult male Sprague-Dawley rats $(\sim 250 \mathrm{~g})$ were anesthetized and placed into a stereotaxic frame. SKIP (5 pg or $500 \mathrm{pg}$ ) or saline vehicle in a volume of $2 \mu \mathrm{l}$ diluted in saline was administered intraparenchymally (supranigral injection) in a single injection via a Hamilton syringe (AP $-5.2 \mathrm{~mm}$, $\mathrm{ML}-1.8, \mathrm{DV}-7.2$; coordinates according to the rat brain atlas of Paxinos and Watson (1998)). Two weeks following the supranigral SKIP injections, the rats were re-anesthetized, placed into a stereotaxic apparatus, and received two unilateral injections of the catecholamine-specific neurotoxin 6-OHDA ( $10 \mu \mathrm{g}$ each in $2 \mu \mathrm{l}$ saline $+0.2 \%$ ascorbic acid $)$ placed into the right striatum to create the partial lesion model $(\mathrm{AP}+1.6 \mathrm{~mm}, \mathrm{ML}-2.4, \mathrm{DV}-4.2$, and $\mathrm{AP}+0.2$, ML - 2.6, DV - 7.0 (Paxinos and Watson, 1998)). The striatal injection of 6-OHDA results in a relatively progressive degeneration of the DAergic nigrostriatal pathway (Sauer and Oertel, 1994; Kirik et al. 1998; Deumens et al. 2002). All animal experiments were performed following an approved protocol by the University of Cincinnati Institutional Animal Care and Use Committee.

Forelimb Asymmetry ("Cylinder”) Test. Animals were tested for motor deficits using the forelimb-use asymmetry test, as described previously (Schallert et al. 2000; Hemmerle et al. 2014; Hu et al. 2017; Kyser et al. 2019). This behavioral test is a simple, noninvasive test of a naturally occurring behavior that is particularly useful in unilateral partial lesion models (Schallert et al. 2000; Lundblad et al. 2005). The procedure was performed under video surveillance and at least an hour into the dark cycle under low-light conditions. Subjects were placed into a clear Plexiglas cylinder (38.1 cm height, $25.4 \mathrm{~cm}$ diameter) and allowed to naturally explore the walls by rearing up and using their forelimbs for weight support. The rats were recorded for a maximum of $5 \mathrm{~min}$ in the cylinder. Videotaped behavior was scored in 
slow motion by an experimenter blind to the condition. A percent limb usage score was calculated using the formula ((contralateral side $+1 / 2$ both)/(ipsilateral side + contralateral side + both $)) \times 100$. A score of $50 \%$ indicates equal use of both forelimbs, whereas scores less than 50\% indicate motor deficits in the contralateral forelimb. Statistical analyses included one-way ANOVA followed by Neuman-Kuels post hoc test.

Immunohistochemistry and Stereology. At 4 weeks postlesion, the animals were sacrificed and processed for immunohistochemistry. Following perfusion with $4 \%$ paraformaldehyde, brain dissection, post-fixation, and cryoprotection, coronal sections through ventral mesencephalon were cut on a sliding microtome at thickness of $50 \mu \mathrm{m}$. Series of free-floating mesencephalic sections were processed for tyrosine hydroxylase (TH; catecholamine biosynthetic enzyme and marker for midbrain dopamine cells) immunocytochemistry, as previously described (Seroogy et al. 1989; Hemmerle et al. 2014). TH immunostained cells were counted in the midbrain (substantia nigra pars compacta; SNpc) using Stereo Investigator 5.05 (MicroBrighfield, Williston, VT) utilizing unbiased stereological techniques, as detailed previously (West, 1993; Hemmerle et al. 2014; Hu et al. 2017). Contours were defined at $2.5 \times$ magnification, and cells were counted at $60 \times$ using the optical fractionator. The random sample sites had a grid size of $170 \times 100$, with a guard zone of $2 \mu \mathrm{m}$. The coefficient of error (CE), as determined using the Gunderson correction, was $\leq 0.10$. The extent of cell loss in the $\mathrm{SNpc}$ was determined by comparing the

\section{A Cell viability (Rotenone, 24hrs)}

\section{B Cell viability (Rotenone, 48hrs)}
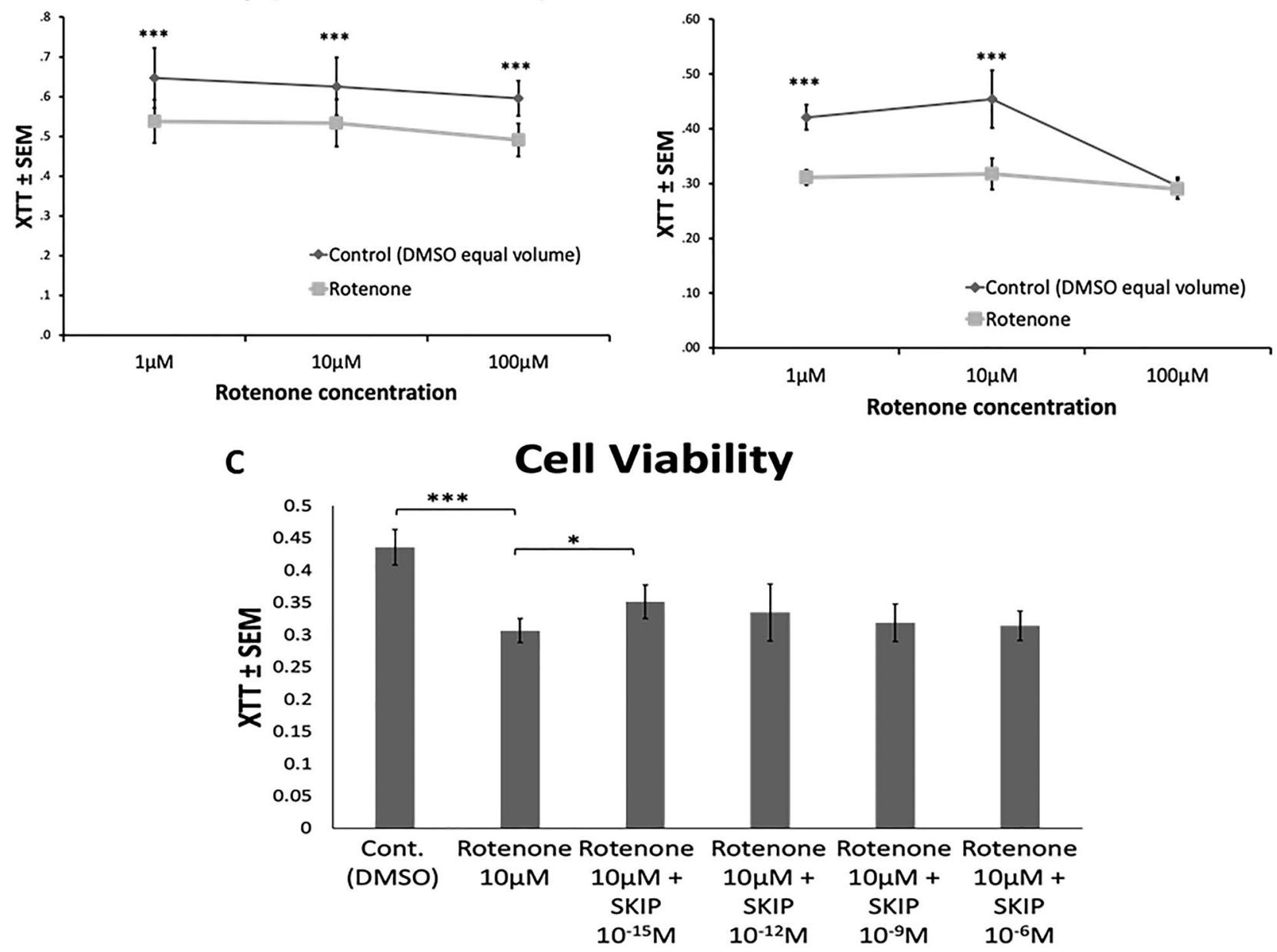

Fig. 1 Rotenone produces limited neuronal cell death, SKIP provides protection. SH-SY5Y cells were plated onto 96-well plates at a concentration of 3000 cells/well and then differentiated to neuronallike cells with retinoic acid $(10 \mu \mathrm{M})$ for 7 days. On the experimental day, cells were treated with 1,10 , and $100 \mu \mathrm{M}$ of rotenone for $24 \mathrm{~h}$ (A) or $48 \mathrm{~h}(\mathbf{B})$. C Treatment with $10 \mu \mathrm{M}$ of rotenone for $48 \mathrm{~h}$ in the presence of increasing SKIP concentrations. Mitochondrial activ- ity measurements (cell viability) were obtained by the XTT colorimetric assay. Statistical analysis was performed by one-way ANOVA, Tukey (A and B) or LSD (C) HSD. Statistical significance is represented within the concentration group ( $\mathbf{A}$ and $\mathbf{B}$ ) or in relation to the rotenone group: "Rotenone $10 \mu \mathrm{M}$ " (C). $n=20 /$ group, $* p<0.05$, $* * * p<0.001$ 
lesioned side to the contralateral unlesioned side. Statistical analyses included one-way ANOVA followed by Neuman-Kuels post hoc test.

\section{Results}

\section{Rotenone Exhibits Minimal Cellular Toxicity in the Cell Viability Assay (XTT); SKIP Provides Limited Protection}

Compared with control (DMSO), rotenone treatment reduced neuronal-like SH-SY5Y cell viability between 15 and $40 \%$, depending on the dose $(1,10,100 \mu \mathrm{M})$ and duration of incubation ( 24 and $48 \mathrm{~h}$ ). This is in line with the "sick but not dead" concept, thereby allowing testing for effects of SKIP (Fig. 1A, B). Surprisingly, the $100 \mu \mathrm{M}, 48$-h rotenone treatment was nontoxic, although apparent consistent toxicity was observed with the lower tested concentrations. To evaluate the protective effect of SKIP against rotenone cytotoxicity, differentiated SHSY5Y cells were exposed to $10 \mu \mathrm{M}$ of rotenone (exhibiting reproducible toxic effects; Fig. 1A, B) with or without SKIP at different concentrations $\left(10^{-15}-10^{-6} \mathrm{M}\right)$ for $48 \mathrm{~h}$. A minor but statistically significant protective effect against rotenone toxicity was observed after treatment with $10^{-15}$ M SKIP (Fig. 1C).

\section{SKIP Blunts Rotenone-Induced Decreases in Neuritic Branch Length and MT Polymerization}

Rotenone was tested for reduction of neuritic length in SHSY5Y cells at two concentrations $(1$ and $10 \mu \mathrm{M})$ and SKIP was added $\left(10^{-15}-10^{-9} \mathrm{M}\right)$, for 2 or $24 \mathrm{~h}$. Whereas a $2-\mathrm{h}$ exposure was either not significant or less effective (data not shown), 24-h rotenone treatment repeatedly reduced branch length by $\sim 50 \%$ (Fig. 2A). While no SKIP protection was observed with SKIP at $10^{-15} \mathrm{M}, 10^{-12} \mathrm{M}$, or $10^{-9} \mathrm{M}$ against $10 \mu \mathrm{M}$ rotenone (24-h exposure period, data not shown), treatment with SKIP at $10^{-15} \mathrm{M}$ or $10^{-9} \mathrm{M}$ provided protection against $1 \mu \mathrm{M}$ rotenone (24-h exposure period; Fig. 2A). The tested $10^{-12}$ M SKIP did not differ from the other tested concentrations.

A Cell Morphology (differentiated SH-SY5Y cells, Rotenone $1 \mu \mathrm{M}, 24 \mathrm{hrs}$ )

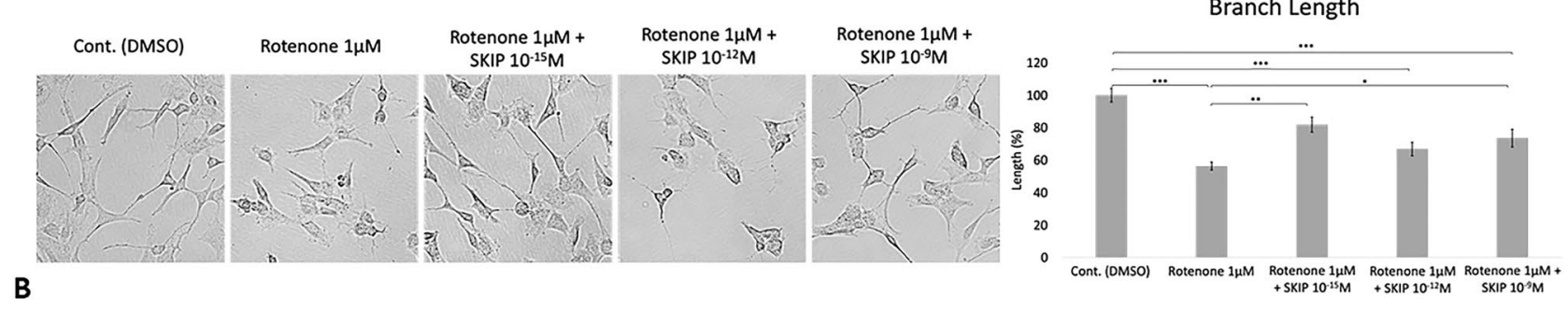

B

Tubulin polymerization assay (differentiated SH-SY5Y cells, Rotenone $10 \mu \mathrm{M}, 24 \mathrm{hrs}$ )

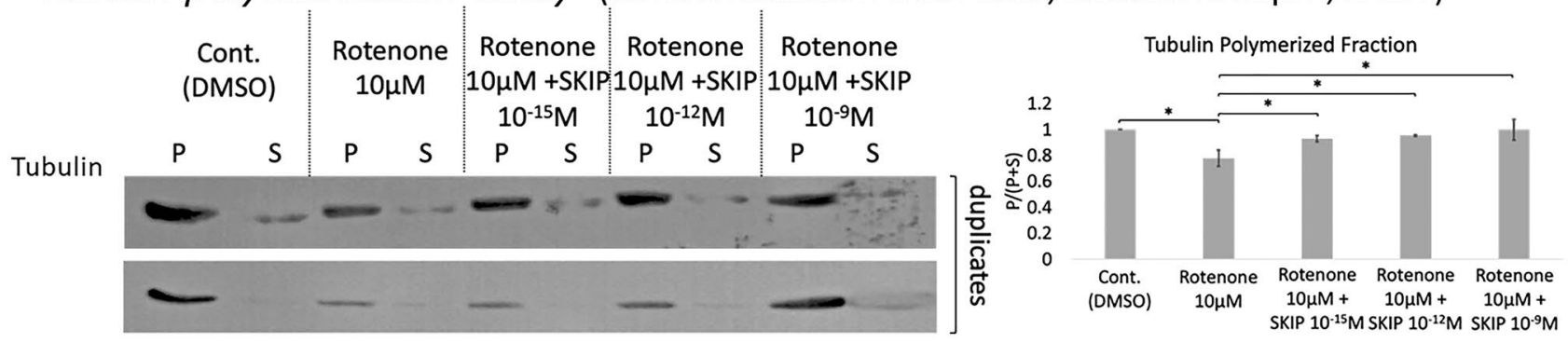

Fig. 2 Rotenone reduces neurite length and polymerized MT content. A Neurite length assessment is described in the "Methods" and the "Results". Confocal microscopy pictures and quantitative analysis are depicted side by side. Statistical analysis was performed by one-way ANOVA, Tukey HSD. Experimental groups tested included: "Rotenone $1 \mu \mathrm{M} 2$ h" group: Cont (DMSO) $n=78$; Rotenone $1 \mu \mathrm{M} n=38$; Rotenone $1 \mu \mathrm{M}+$ SKIP $10^{-15} \mathrm{M} n=71$; Rotenone $1 \mu \mathrm{M}+\mathrm{SKIP}$ $10^{-12}$ M $n=56$; Rotenone $1 \mu \mathrm{M}+\operatorname{SKIP} 10^{-9} \mathrm{M} n=74$. "Rotenone $1 \mu \mathrm{M} 24$ h" group: Cont (DMSO) $n=135$; Rotenone $1 \mu \mathrm{M} n=122$; Rotenone $1 \mu \mathrm{M}+\mathrm{SKIP} 10^{-15} \mathrm{M} n=49$; Rotenone $1 \mu \mathrm{M}+\mathrm{SKIP}$
$10^{-12} \mathrm{M} n=54$; Rotenone $1 \mu \mathrm{M}+$ SKIP $10^{-9} \mathrm{M} n=98$. "Rotenone $10 \mu \mathrm{M} 2$ h" group: Cont (DMSO) $n=215$; Rotenone $10 \mu \mathrm{M} n=190$; Rotenone $10 \mu \mathrm{M}+$ SKIP $10^{-15} \mathrm{M} n=178$; Rotenone $10 \mu \mathrm{M}+\mathrm{SKIP}$ $10^{-12} \mathrm{M} n=162$; Rotenone $10 \mu \mathrm{M}+$ SKIP $10^{-9} \mathrm{M} n=185$. "Rotenone $10 \mu \mathrm{M} 24$ h" group: Cont (DMSO) $n=176$; Rotenone $10 \mu \mathrm{M} n=133$; Rotenone $10 \mu \mathrm{M}+\operatorname{SKIP} 10^{-15} \mathrm{M} n=110$; Rotenone $10 \mu \mathrm{M}+\mathrm{SKIP}$ $10^{-12} \mathrm{M} n=111$; Rotenone $10 \mu \mathrm{M}+\operatorname{SKIP} 10^{-9} \mathrm{M} n=114$. B Polymerized vs. soluble tubulin/MT assay is described in the "Methods" and "Results" section. Data were statistically analyzed by one-way ANOVA, LSD HSD ( $n=2$ /group). $* p<0.05$ 


\section{SKIP Protects Against Rotenone-Induced Decreases in MT Polymerization}

After assessment of cell morphology (Fig. 2A), cells were harvested, and lysates were separated into polymerized (P) and soluble $(\mathrm{S})$ protein fractions. Equal aliquots of each pair ( $\mathrm{P}$ and $\mathrm{S}$ ) were resolved on adjacent lanes by SDS polyacrylamide gel electrophoresis. The blots were probed with tubulin antibodies. The intensity of each band was quantified by Fiji and ratios were calculated by dividing the intensity value of the pelleted band over the sum of intensity values of polymerized and soluble bands [P/ $(\mathrm{S}+\mathrm{P})$ ]. Results after $24-\mathrm{h}$ incubation with $10 \mu \mathrm{M}$ rotenone indicated an $\sim 30 \%$ significant decrease in the relative content of polymerized MT, which was completely reversed by SKIP treatment, at all tested concentrations $\left(10^{-15}-10^{-9} \mathrm{M}\right.$; Fig. 2B). Notably, in each series of experiments, we used different concentrations of rotenone to test the optimal concentration at which the specific experiment would work in the best manner. Only statistically significant results are presented. The potent rotenone and
SKIP concentrations vary between experiments, probably, because of different vulnerability of the tested cell systems to the tested drugs.

\section{Rotenone Decreases MT Dynamics; SKIP Protects MT Speed}

We aimed to evaluate the effect of rotenone on MT dynamics and of the possible protective activity of SKIP against rotenone-induced MT disruption. Differentiated neuronallike neuroblastoma cells (NIE-115 cells) were subjected to transient transfection with an expression plasmid encoding EB3 protein, tagged to RFP (EB3-RFP). Indeed, live cell imaging of MT dynamics requires cell transfection with EB3-RFP expressing plasmid (Ivashko-Pachima et al. 2017). Here, we chose to use the easily transfected mouse neuroblastoma N1E-115 cells (Ivashko-Pachima et al. 2017, 2019b, 2021a; Ivashko-Pachima and Gozes 2019a, 2021b; Grigg et al. 2020).

Single-cell time-lapse imaging allowed us to evaluate the effect of rotenone on MT dynamics by tracking EB3-RFP

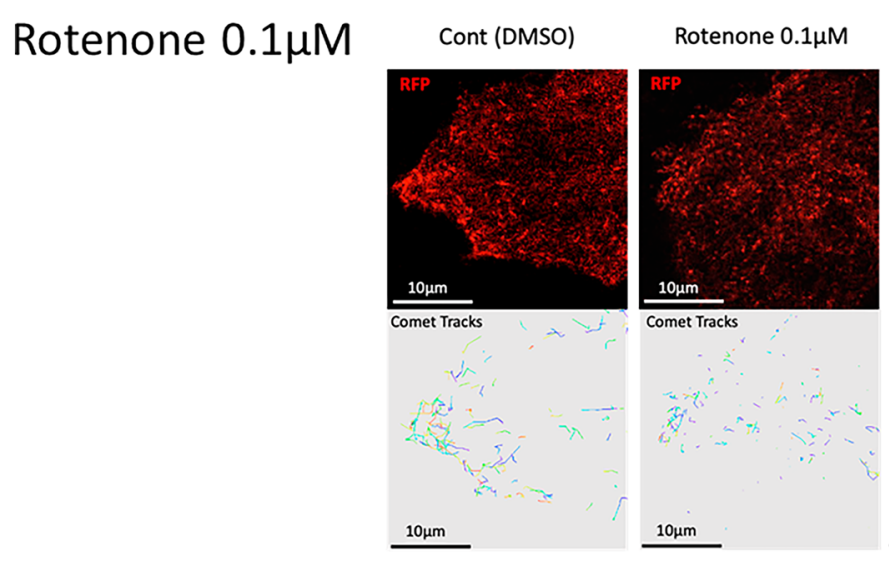

Track Length Rotenone $0.1 \mu \mathrm{M}$

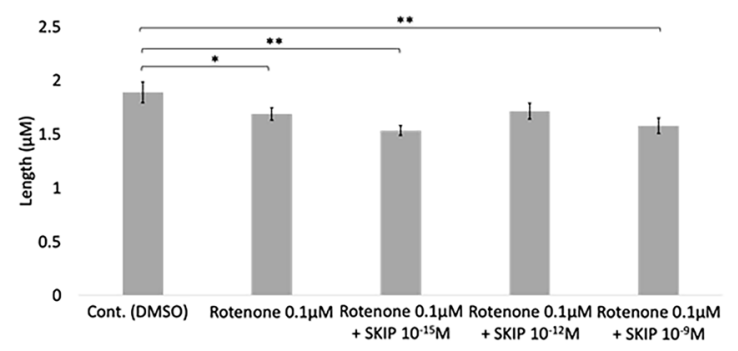

Rotenone $0.1 \mu \mathrm{M}+$ SKIP $10^{-15} \mathrm{M}$
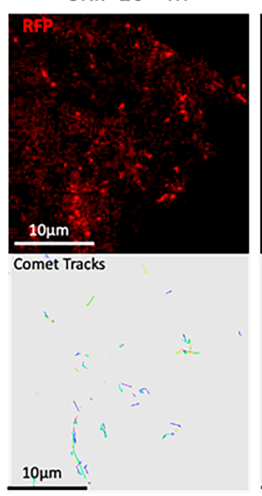

Rotenone $0.1 \mu \mathrm{M}+$ SKIP $10^{-12} \mathrm{M}$

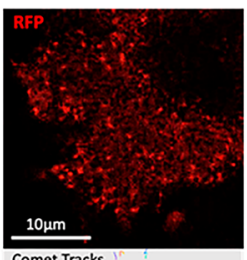

Comet Tracks

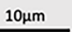

Rotenone $0.1 \mu \mathrm{M}+$ SKIP $10^{-9} \mathrm{M}$

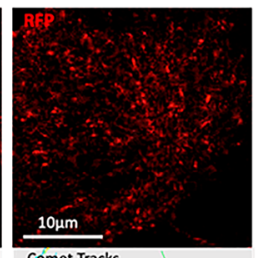

Cómet Tracks

Comet Speed Rotenone 0.1 $\mu \mathrm{M}$

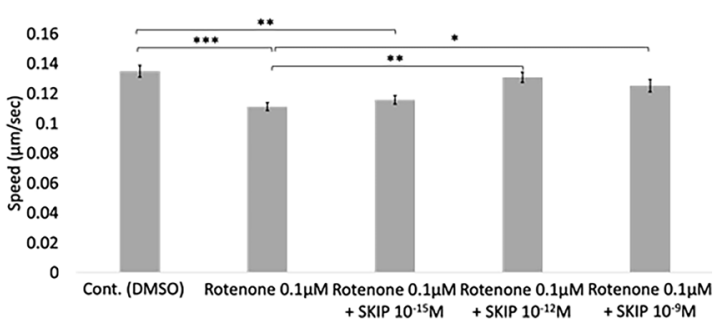

Fig. 3 Rotenone reduces MT dynamics, SKIP protects. Live cell imaging evaluations were carried out as described in the "Methods" and the "Results", live imaging was conducted for $2 \mathrm{~h}$, and results with $0.1 \mu \mathrm{M}$ rotenone are shown: upper panel—graphic calculations, lower panel-confocal imaging and track tracing (Ivashko-Pachima et al. 2017). Statistical analysis was performed by one-way ANOVA, Tukey HSD. Group used included: "Rotenone $10 \mu \mathrm{M}$ " group: Cont (DMSO) $n=27$; Rotenone $10 \mu \mathrm{M} n=10$; Rotenone $10 \mu \mathrm{M}+\mathrm{SKIP}$
$10^{-15} \mathrm{M} n=12$; Rotenone $10 \mu \mathrm{M}+\mathrm{SKIP} 10^{-12} \mathrm{M} n=13$; Rotenone $10 \mu \mathrm{M}+$ SKIP $10^{-9} \mathrm{M} n=10$. "Rotenone $1 \mu \mathrm{M}$ " group: Cont (DMSO) $n=29$; Rotenone $1 \mu \mathrm{M} n=14$; Rotenone $1 \mu \mathrm{M}+\mathrm{SKIP}$ $10^{-15} \mathrm{M} n=11$; Rotenone $1 \mu \mathrm{M}+\mathrm{SKIP} 10^{-12} \mathrm{M} n=11$; Rotenone $1 \mu \mathrm{M}+\operatorname{SKIP} 10^{-9} \mathrm{M} n=12$. "Rotenone $0.1 \mu \mathrm{M}$ " group: Cont (DMSO) $n=17$; Rotenone $0.1 \mu \mathrm{M} n=17$; Rotenone $0.1 \mu \mathrm{M}+\mathrm{SKIP}$ $10^{-15} \mathrm{M} n=14$; Rotenone $0.1 \mu \mathrm{M}+\operatorname{SKIP} 10^{-12} \mathrm{M} n=15$; Rotenone $0.1 \mu \mathrm{M}+\operatorname{SKIP} 10^{-9} \mathrm{M} n=14 . * p<0.05,{ }^{* *} p<0.01$, *** $p<0.001$ 
A

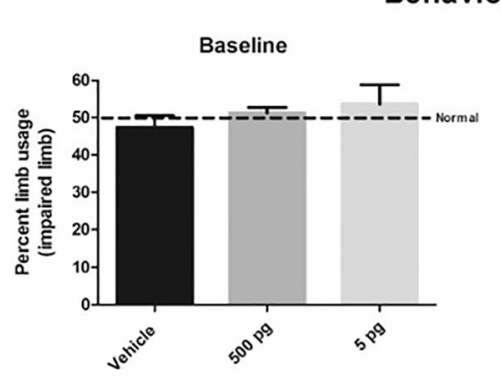

Behavior Scores

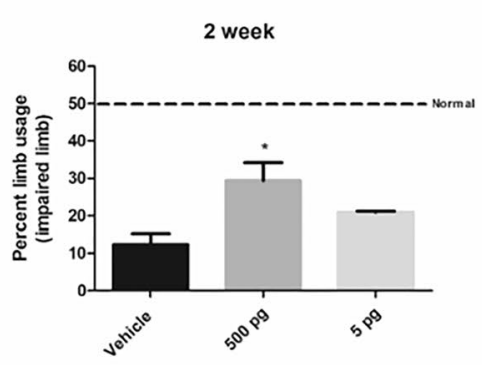

TH SNpc Cell Counts

B

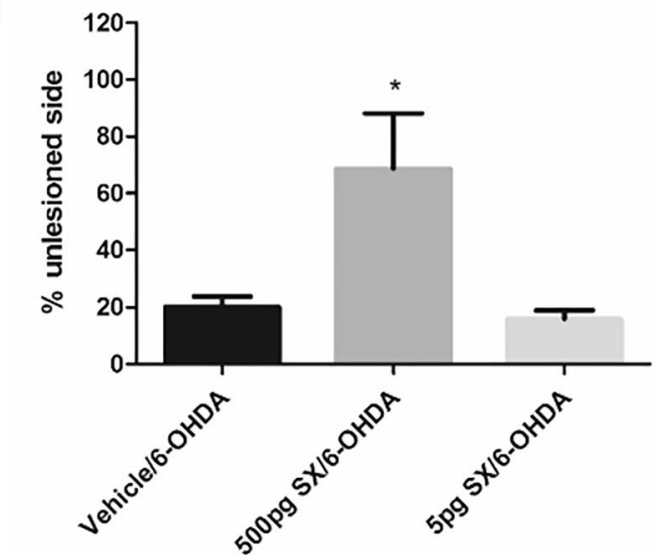

Fig. 4 SKIP partially ameliorates 6-OHDA lesion-induced deficits. A Behavioral asymmetry scores in the cylinder test. At baseline ( 2 weeks after supranigral SKIP or vehicle administration, but before striatal 6-OHDA injection), all 3 groups exhibited equal usage of each forelimb (left panel). However, at 2 weeks following 6-OHDA lesions, the group pretreated with 500 pg SKIP maintained increased use of the impaired forelimb compared to the groups pretreated with vehicle or 5 pg SKIP (right panel). B Stereological cell counts in the SNpc show that TH cell loss was significantly attenuated in the rats pretreated with $500 \mathrm{pg}$ SKIP compared to those pretreated with $5 \mathrm{pg}$ SKIP or vehicle. TH, tyrosine hydroxylase; $\mathrm{SNpc}$, substantia nigra pars compacta; SX, SKIP. $n=3-4$ /group. $* p<0.05$

improved impaired limb usage (Fig. 4A). Similarly, stereological cell counts of surviving nigral TH-positive neurons at 4 weeks post-6-OHDA lesion revealed considerable cell loss in the vehicle-treated rats. In contrast, pretreatment of lesioned rats with $500 \mathrm{pg}$ (but not $5 \mathrm{pg}$ ) SKIP significantly and substantially increased $\mathrm{TH}+$ cell survival in the $\mathrm{SNpc}$ (Fig. 4B).

\section{Discussion}

The association of the pesticide rotenone and increased toxic dopaminergic metabolites has been shown before (Goldstein et al. 2015). Here, through the prism of neuroprotection, we focused on the MT-associated SKIP/rotenone and DAergic system neurotoxicity. We showed that $10^{-15} \mathrm{M}$ SKIP protected against 48 -h induced $10 \mu \mathrm{M}$ rotenone toxicity, measured at the level of cellular viability (mitochondrial activity). Interestingly, high concentrations of rotenone $(100 \mu \mathrm{M})$ were less toxic to SH-SY5Y cells, exhibiting a bell-shape toxicity effect, in agreement with the SKIP protection curve. Such a neuroprotective bell-shaped dose-response curve with a peak at $10^{-15} \mathrm{M}$ was also seen before for NAP (Bassan et al. 1999). Neurite outgrowth also showed a peak at $10^{-15} \mathrm{M}$ SKIP concentrations, although higher concentrations were effective as well, consonant with previous studies on NAP (Smith-Swintosky et al. 2005). MT comet speed required slightly higher concentrations, suggesting differential target engagements/downstream mechanisms. In this respect, SKIP has been shown previously to enhance ADNP-dependent axonal transport (Amram et al. 2016) and to accelerate tau-MT interaction, through EB1/3 of 500 pg SKIP (but not the lower 5 pg dose) significantly 
(Ivashko-Pachima and Gozes, 2019a). These data are in agreement with NAP protecting MT-dependent axonal transport (Jouroukhin et al. 2013) and accelerating tau-MT interaction (Ivashko-Pachima et al. 2017). The converging protection of SKIP against MT disruption and DAergic-related toxicity was observed here in vivo, in an animal model of 6-OHDA-induced neurotoxicity. Interestingly, protection of the DAergic system in association with protection against tauopathy (Gozes et al. 2009) was previously suggested for NAP in another motor disease, amyotrophic lateral sclerosis (Jouroukhin et al. 2013). Moreover, as pointed out in the "Introduction" section, the ADNP system is dysregulated in PD (Chu et al. 2016).

This is the first in vivo description of SKIP protection outside of the ADNP syndrome (Amram et al. 2016), suggesting a broader range of activity/efficacy. The acute nature of the injury allows acute treatment, but with extensive pharmacodynamic properties (i.e., seeing a protective effect two weeks or even more than that, after administration). This is in accord with our NAP studies in animal models: e.g., head trauma (Beni-Adani et al. 2001; Romano et al. 2002), including pretreatment with NAP (Zaltzman et al. 2003)) and stroke (Leker et al. 2002), as well as the PD models mentioned in the "Introduction" and also in human clinical trials. Thus, in clinical trials in amnestic mild cognitive impairment (a precursor to Alzheimer's disease), NAP administration showed efficacy even 4 weeks after cessation of treatment, suggesting an extensive pharmacodynamics effect (Gozes et al. 2009).

The present results using a pretreatment paradigm are encouraging as they demonstrate the neuroprotective potential of SKIP in a well-established model of PD. However, future experiments should examine posttreatment paradigms to be more clinically relevant, alternate routes of SKIP administration (i.e., intranasal; Fleming et al. 2011; Magen et al. 2014; Bermudez et al. 2019), higher doses of SKIP, and additional PD animal models. Future studies should also look at modified SKIP (Ivashko-Pachima and Gozes 2019a) as well as drug bioavailability and additional PD/ MT-related models, toward clinical development. Lastly, we have previously shown SKIP + NAP interactions. Thus, SKIP enhanced NAP-target (EB3) interaction (affinity column chromatography) and the two peptides showed direct interaction (nuclear magnetic resonance spectroscopy) (Amram et al. 2016). These previous results are suggestive of additive or synergistic effects for SKIP + NAP, which should be further explored.

Acknowledgements The expert contribution of Dr. Ann Hemmerle to the in vivo experiments is gratefully acknowledged.

Funding This work was partially supported by the following grants (IG): European Research Area Network (ERA-NET) Neuron ADNPinMED, the US-Israel Binational Science Foundation - US National Science Foundation (BSF-NSF 2016746), the Alberto Moscona Nisim
(AMN) Foundation for the Advancement of Science, Art and Culture in Israel, as well as by Drs. Ronith and Armand Stemmer and Arthur Gerbi (French Friends of Tel Aviv University) and Anne and Alex Cohen (Canadian Friends of Tel Aviv University). YI-P was supported in part by the Aufzien Family Center for the Prevention and Treatment of Parkinson's Disease (APPD) postdoctoral fellowship. KBS was supported in part by the Kerman Family Fund, the Selma Schottenstein Harris Lab for Research in Parkinson's, the Gardner Family Center for Parkinson's Disease and Movement Disorders, and the Parkinson's Disease Support Network of Ohio, Kentucky and Indiana.

\section{Declarations}

Conflict of Interest NAP (CP201) use is under patent protection (US patent nos. US7960334, US8618043, US10912819) and provisional patent applications; SKIP derivatives are under a composition of matter US patent, US10822375, Ramot at Tel Aviv University (IG).

\section{References}

Amram N, Hacohen-Kleiman G, Sragovich S, Malishkevich A, Katz J, Touloumi O, Lagoudaki R, Grigoriadis NC, Giladi E, Yeheskel A, Pasmanik-Chor M, Jouroukhin Y, Gozes I (2016) Sexual divergence in microtubule function: the novel intranasal microtubule targeting SKIP normalizes axonal transport and enhances memory. Mol Psychiatry 21:1467-1476

Bassan M, Zamostiano R, Davidson A, Pinhasov A, Giladi E, Perl O, Bassan H, Blat C, Gibney G, Glazner G, Brenneman DE, Gozes I (1999) Complete sequence of a novel protein containing a femtomolar-activity-dependent neuroprotective peptide. $\mathrm{J}$ Neurochem 72:1283-1293

Beni-Adani L, Gozes I, Cohen Y, Assaf Y, Steingart RA, Brenneman DE, Eizenberg O, Trembolver V, Shohami E (2001) A peptide derived from activity-dependent neuroprotective protein (ADNP) ameliorates injury response in closed head injury in mice. J Pharmacol Exp Ther 296:57-63

Bermudez ML, Seroogy KB, Genter MB (2019) Evaluation of carnosine intervention in the Thy1-aSyn mouse model of Parkinson's disease. Neuroscience 411:270-278

Burke WJ, Li SW, Williams EA, Nonneman R, Zahm DS (2003) 3,4-Dihydroxyphenylacetaldehyde is the toxic dopamine metabolite in vivo: implications for Parkinson's disease pathogenesis. Brain Res 989:205-213

Choi W-S, Palmiter RD, Xia Z (2011) Loss of mitochondrial complex I activity potentiates dopamine neuron death induced by microtubule dysfunction in a Parkinson's disease model. J Cell Biol 192:873-82

Chu Y, Morfini GA, Kordower JH (2016) Alterations in activitydependent neuroprotective protein in sporadic and experimental Parkinson's disease. J Parkinsons Dis 6:77-97

Deumens R, Blokland A, Prickaerts J (2002) Modeling Parkinson's disease in rats: an evaluation of 6-OHDA lesions of the nigrostriatal pathway. Exp Neurol 175:303-317

Esteves AR, Gozes I, Cardoso SM (2014) The rescue of microtubuledependent traffic recovers mitochondrial function in Parkinson's disease. Biochem Biophys Acta 1842:7-21

Fleming SM, Mulligan CK, Richter F, Mortazavi F, Lemesre V, Frias C, Zhu C, Stewart A, Gozes I, Morimoto B, Chesselet MF (2011) A pilot trial of the microtubule-interacting peptide (NAP) in mice overexpressing alpha-synuclein shows improvement in motor function and reduction of alpha-synuclein inclusions. Mol Cell Neurosci 46:597-606 
Goldstein DS, Sullivan P, Cooney A, Jinsmaa Y, Kopin IJ, Sharabi Y (2015) Rotenone decreases intracellular aldehyde dehydrogenase activity: implications for the pathogenesis of Parkinson's disease. J Neurochem 133:14-25

Goldstein DS, Sullivan P, Holmes C, Miller GW, Alter S, Strong R, Mash DC, Kopin IJ, Sharabi Y (2013) Determinants of buildup of the toxic dopamine metabolite DOPAL in Parkinson's disease. J Neurochem 126:591-603

Gozes I (2020) The ADNP Syndrome and CP201 (NAP) Potential and hope. Front Neurol 11:608444

Gozes I, Morimoto BH, Tiong J, Fox A, Sutherland K, Dangoor D, Holser-Cochav M, Vered K, Newton P, Aisen PS, Matsuoka Y, van Dyck CH, Thal L (2005) NAP: research and development of a peptide derived from activity-dependent neuroprotective protein (ADNP). CNS Drug Rev 11:353-368

Gozes I, Stewart A, Morimoto B, Fox A, Sutherland K, Schmeche D (2009) Addressing Alzheimer's disease tangles: from NAP to AL-108. Curr Alzheimer Res 6:455-460

Grigg I, Ivashko-Pachima Y, Hait TA, Korenkova V, Touloumi O, Lagoudaki R, Van Dijck A, Marusic Z, Anicic M, Vukovic J, Kooy RF, Grigoriadis N, Gozes I (2020) Tauopathy in the young autistic brain: novel biomarker and therapeutic target. Transl Psychiatry 10:228

Hacohen-Kleiman G, Sragovich S, Karmon G, Gao AYL, Grigg I, Pasmanik-Chor M, Le A, Korenkova V, McKinney RA, Gozes I (2018) Activity-dependent neuroprotective protein deficiency models synaptic and developmental phenotypes of autism-like syndrome. J Clin Investig 128:4956-4969

Hadar A, Kapitansky O, Ganaiem M, Sragovich S, Lobyntseva A, Giladi E, Yeheskel A, Avitan A, Vatine GD, Gurwitz D, Ivashko-Pachima Y, Gozes I (2021) Introducing ADNP and SIRT1 as new partners regulating microtubules and histone methylation. Mol Psychiatry

Hemmerle AM, Dickerson JW, Herman JP, Seroogy KB (2014) Stress exacerbates experimental Parkinson's disease. Mol Psychiatry 19:638-640

Hu J, Ferchmin PA, Hemmerle AM, Seroogy KB, Eterovic VA, Hao J (2017) 4R-Cembranoid improves outcomes after 6-hydroxydopamine challenge in both in vitro and in vivo models of Parkinson's disease. Front Neurosci 11:272

Ivashko-Pachima Y, Gozes I (2019a) A novel microtubule-Tau association enhancer and neuroprotective drug candidate: Ac-SKIP. Front Cell Neurosci 13:435

Ivashko-Pachima Y, Maor-Nof M, Gozes I (2019b) NAP (davunetide) preferential interaction with dynamic 3-repeat Tau explains differential protection in selected tauopathies. PloS one 14:e0213666.

Ivashko-Pachima Y, Hadar A, Grigg I, Korenkova V, Kapitansky O, Karmon G, Gershovits M, Sayas CL, Kooy RF, Attems J, Gurwitz D, Gozes I (2021a) Discovery of autism/intellectual disability somatic mutations in Alzheimer's brains: mutated ADNP cytoskeletal impairments and repair as a case study. Mol Psychiatry 26:1619-1633

Ivashko-Pachima Y, Gozes I (2021b) Activity-dependent neuroprotective protein (ADNP)-end-binding protein (EB) interactions regulate microtubule dynamics toward protection against tauopathy. Prog Mol Biol Transl Sci 177:65-90

Ivashko-Pachima Y, Sayas CL, Malishkevich A, Gozes I (2017) ADNP/NAP dramatically increase microtubule end-binding protein-Tau interaction: a novel avenue for protection against tauopathy. Mol Psychiatry 22:1335-1344

Jouroukhin Y, Ostritsky R, Assaf Y, Pelled G, Giladi E, Gozes I (2013) NAP (davunetide) modifies disease progression in a mouse model of severe neurodegeneration: protection against impairments in axonal transport. Neurobiol Dis 56:79-94

Kirik D, Rosenblad C, Bjorklund A (1998) Characterization of behavioral and neurodegenerative changes following partial lesions of the nigrostriatal dopamine system induced by intrastriatal 6-hydroxydopamine in the rat. Exp Neurol 152:259-277

Kish SJ, Shannak K, Hornykiewicz O (1988) Uneven pattern of dopamine loss in the striatum of patients with idiopathic Parkinson's disease. Pathophysiologic and clinical implications. $\mathrm{N}$ Engl J Med 318:876-880

Kyser TL, Dourson AJ, McGuire JL, Hemmerle AM, Williams MT, Seroogy KB (2019) Characterization of motor and non-motor behavioral alterations in the $\mathrm{Dj}-1$ (PARK7) knockout rat. J Mol Neurosci 69:298-311

Leker RR, Teichner A, Grigoriadis N, Ovadia H, Brenneman DE, Fridkin M, Giladi E, Romano J, Gozes I (2002) NAP, a femtomolar-acting peptide, protects the brain against ischemic injury by reducing apoptotic death. Stroke 33:1085-1092

Lundblad M, Usiello A, Carta M, Hakansson K, Fisone G, Cenci MA (2005) Pharmacological validation of a mouse model of 1-DOPA-induced dyskinesia. Exp Neurol 194:66-75

Magen I, Ostritsky R, Richter F, Zhu C, Fleming SM, Lemesre V, Stewart AJ, Morimoto BH, Gozes I, Chesselet MF (2014) Intranasal NAP (davunetide) decreases tau hyperphosphorylation and moderately improves behavioral deficits in mice overexpressing alpha-synuclein. Pharmacol Res Perspect 2(5): 00065

Mandel S, Rechavi G, Gozes I (2007) Activity-dependent neuroprotective protein (ADNP) differentially interacts with chromatin to regulate genes essential for embryogenesis. Dev Biol 303:814-824

Melo TQ, van Zomeren KC, Ferrari MF, Boddeke HW, Copray JC (2017) Impairment of mitochondria dynamics by human A53T alpha-synuclein and rescue by NAP (davunetide) in a cell model for Parkinson's disease. Exp Brain Res 235:731-742

Offen D, Sherki Y, Melamed E, Fridkin M, Brenneman DE, Gozes I (2000) Vasoactive intestinal peptide (VIP) prevents neurotoxicity in neuronal cultures: relevance to neuroprotection in Parkinson's disease. Brain Res 854:257-262

Oz S, Kapitansky O, Ivashco-Pachima Y, Malishkevich A, Giladi E, Skalka N, Rosin-Arbesfeld R, Mittelman L, Segev O, Hirsch JA, Gozes I (2014) The NAP motif of activity-dependent neuroprotective protein (ADNP) regulates dendritic spines through microtubule end binding proteins. Mol Psychiatry 19:1115-1124

Passmore JB, Pinho S, Gomez-Lazaro M, Schrader M (2017) The respiratory chain inhibitor rotenone affects peroxisomal dynamics via its microtubule-destabilising activity. Histochem Cell Biol 148:331-341

Paxinos G, Watson C (1998) The rat brain in stereotaxic coordinates. Academic Press, New York

Pinhasov A, Mandel S, Torchinsky A, Giladi E, Pittel Z, Goldsweig AM, Servoss SJ, Brenneman DE, Gozes I (2003) Activitydependent neuroprotective protein: a novel gene essential for brain formation. Brain Res Dev Brain Res 144:83-90

Ren Y, Feng J (2007) Rotenone selectively kills serotonergic neurons through a microtubule-dependent mechanism. J Neurochem 103:303-311

Ren Y, Liu W, Jiang H, Jiang Q, Feng J (2005) Selective vulnerability of dopaminergic neurons to microtubule depolymerization. J Biol Chem 280:34105-34112

Ren Y, Zhao J, Feng J (2003) Parkin binds to alpha/beta tubulin and increases their ubiquitination and degradation. J Neurosci 23:3316-3324

Romano J, Beni-Adani L, Nissenbaum OL, Brenneman DE, Shohami E, Gozes I (2002) A single administration of the peptide NAP induces long-term protective changes against the consequences of head injury: gene Atlas array analysis. J Mol Neurosci 18:37-45

Sauer H, Oertel WH (1994) Progressive degeneration of nigrostriatal dopamine neurons following intrastriatal terminal lesions with 
6-hydroxydopamine: a combined retrograde tracing and immunocytochemical study in the rat. Neuroscience 59:401-415

Schallert T, Fleming SM, Leasure JL, Tillerson JL, Bland ST (2000) CNS plasticity and assessment of forelimb sensorimotor outcome in unilateral rat models of stroke, cortical ablation, parkinsonism and spinal cord injury. Neuropharmacology 39:777-787

Schapira AH. (2008) Mitochondria in the aetiology and pathogenesis of Parkinson's disease. Lancet Neurol 7:97-109

Schindelin J, Arganda-Carreras I, Frise E, Kaynig V, Longair M, Pietzsch T, Preibisch S, Rueden C, Saalfeld S, Schmid B, Tinevez JY, White DJ, Hartenstein V, Eliceiri K, Tomancak P, Cardona A (2012) Fiji: an open-source platform for biological-image analysis. Nat Methods 9:676-682

Seroogy KB, Dangaran K, Lim S, Haycock JW, Fallon JH (1989) Ventral mesencephalic neurons containing both cholecystokinin- and tyrosine hydroxylase-like immunoreactivities project to forebrain regions. J Comp Neurol 279:397-414

Smith-Swintosky VL, Gozes I, Brenneman DE, D'Andrea MR, PlataSalaman CR (2005) Activity-dependent neurotrophic factor-9 and NAP promote neurite outgrowth in rat hippocampal and cortical cultures. J Mol Neurosci 25:225-238

Sragovich S, Malishkevich A, Piontkewitz Y, Giladi E, Touloumi O, Lagoudaki R, Grigoriadis N, Gozes I (2019) The autism/ neuroprotection-linked ADNP/NAP regulate the excitatory glutamatergic synapse. Transl Psychiatry 9:2
Srivastava P, Panda D (2007) Rotenone inhibits mammalian cell proliferation by inhibiting microtubule assembly through tubulin binding. FEBS J 274:4788-4801

Vulih-Shultzman I, Pinhasov A, Mandel S, Grigoriadis N, Touloumi O, Pittel Z, Gozes I (2007) Activity-dependent neuroprotective protein snippet NAP reduces tau hyperphosphorylation and enhances learning in a novel transgenic mouse model. J Pharmacol Exp Ther 323:438-449

West MJ (1993) New stereological methods for counting neurons. Neurobiol Aging 14:275-285

Zaltzman R, Beni SM, Giladi E, Pinhasov A, Steingart RA, Romano J, Shohami E, Gozes I (2003) Injections of the neuroprotective peptide NAP to newborn mice attenuate head-injury-related dysfunction in adults. NeuroReport 14:481-484

Zamostiano R, Pinhasov A, Gelber E, Steingart RA, Seroussi E, Giladi E, Bassan M, Wollman Y, Eyre HJ, Mulley JC, Brenneman DE, Gozes I (2001) Cloning and characterization of the human activity-dependent neuroprotective protein. J Biol Chem 276:708-714

Publisher's Note Springer Nature remains neutral with regard to jurisdictional claims in published maps and institutional affiliations. 